\title{
Relações entre interesses, personalidade e habilidades cognitivas: um estudo com adolescentes ${ }^{1}$
}

\author{
Maiana Farias Oliveira Nunes ${ }^{2}$ - Universidade São Francisco, Itatiba, Brasil \\ Ana Paula Porto Noronha - Universidade São Francisco, Itatiba, Brasil
}

\begin{abstract}
Resumo
Este estudo objetivou analisar as relações entre interesses, personalidade e inteligência em adolescentes. Participaram 211 alunos do ensino médio do estado do Paraná, com média de idade de 16,5 (DP=0,5). Foram utilizadas a Escala de Aconselhamento Profissional e o Self Directed Search para medida dos interesses; a Bateria Fatorial de Personalidade e a Bateria de Provas de Raciocínio. Foi realizada uma análise fatorial com os escores dos quatro testes supracitados, e uma solução de seis fatores indicou maior coerência teórica e prática para compreender as associações entre traços de personalidade, interesses e habilidades cognitivas. Os escores de interesse nos dois testes estiveram mais relacionados, juntamente com alguns fatores de personalidade, com exceção dos traços neuroticismo e realização, enquanto as provas de inteligência não se agruparam com interesses ou personalidade. Discute-se a utilidade de testes que medem os construtos interesses, personalidade e inteligência em orientação profissional.

Palavras-chave: avaliação psicológica; orientação vocacional; traços de personalidade; interesses profissionais; inteligência.
\end{abstract}

\section{Relationships between interests, personality and cognitive abilities: a study with adolescents}

\begin{abstract}
This study aimed at analyzing the relationships between interests, personality and intelligence with adolescents. 211 High School students participated in this research, from Paraná, mean age 16.5 years old (SD=0.5). Students answered the "Escala de Aconselhamento Profissional" and Self Directed Search for measuring interests, the "Bateria Fatorial de Personalidade", for personality assessment and the "Bateria de Provas de Raciocínio", for cognitive assessment. Factor analysis was conducted with scores of the four tests mentioned, and a six-factor solution was more coherent in theoretical and practical terms, in order to help understanding relationships between personality traits, interests and cognitive abilities. The interests' scores on the two tests were more related to each other, including some personality aspects, except for neuroticism and conscientiousness. Intelligence tests were grouped in a single factor, showing no strong relation to interests or personality. The importance of using tests for measuring interests, personality and intelligence is discussed, considering vocational guidance context.

Keywords: psychological assessment; vocational guidance; personality traits; vocational interests; intelligence.
\end{abstract}

\section{Introdução}

A avaliação em Orientação Profissional/ Vocacional (OPV) geralmente envolve a análise de construtos como interesses, necessidades de trabalho, valores, maturidade e indecisão profissional. $\mathrm{O}$ uso de testes nesse contexto busca fornecer informações que deverão auxiliar na ampliação do autoconhecimento ou na tomada de decisão profissional (American Educational Research Association, American Psychological Association \& National Council on Measurement in Education - APA, AERA, NCME, 1999).

Ao discutir o futuro da pesquisa em avaliação com foco em OPV, Betz e Borgen (2000) sugerem que os três focos de ação principais deveriam ser a integração dos construtos interesses, auto-eficácia e personalidade.
No Brasil, os construtos inteligência e personalidade receberam maior atenção de pesquisadores que apresentaram painéis nos congressos de avaliação psicológica (Joly, Silva, Nunes \& Souza, 2007), sendo que a mesma consideração vale para os trabalhos apresentados em mesas redondas deste congresso, com uma pequena participação de estudos $(3,1 \%)$ sobre os interesses profissionais (Noronha \& Nunes, 2008). Essas duas pesquisas ressaltam a pequena participação da temática OPV nos congressos de avaliação psicológica, seja com pôsteres ou apresentações em mesas redondas (Joly \& cols, 2007; Noronha \& Nunes, 2008).

Sobre o uso de testes na área de OPV, Noronha e cols (2006) destacam que o mesmo é restrito, o que foi verificado em uma pesquisa que analisou as temáticas de teses e dissertações produzidas no Brasil, com foco em trabalhos

\footnotetext{
${ }^{1}$ As autoras agradecem o apoio da Capes e do CNPq.

${ }^{2}$ Endereço para correspondência:

Universidade São Francisco - Programa de Pós Graduação Stricto Sensu em Psicologia

Rua Alexandre Rodrigues Barbosa, 45 - 13251-900 - Itatiba-SP, Brasil.

E-mail: maiananunes@mac.com
} 
relacionados à OPV. Uma hipótese para esse achado é que, conforme apontado por Sparta, Bardagi e Teixeira (2006), alguns modelos teóricos de avaliação em OPV não recomendam o uso de testes em situações de aconselhamento de carreira, o que talvez tenha desestimulado pesquisadores da área a utilizar métodos sistemáticos de avaliação.

Vale destacar que, nacionalmente, OPV encontra-se em momento de transformações, tal como pontuado por Sparta, Bardagi e Teixeira (2006). O conteúdo priorizado nesses processos, assim como a forma de realização, tem sofrido modificações em razão das demandas impostas pelas novas configurações do mundo do trabalho e das expectativas dos indivíduos diante disso. A ideia é corroborada pelos achados de Melo-Silva (1999) e Noronha e Ambiel (2006). A primeira enfatizou o crescimento da área a partir da década de 1990, enquanto os últimos alertaram que ainda é necessário o desenvolvimento de instrumentos de avaliação para uso neste contexto. Por fim, sobre as carências evidenciadas na área de OPV no Brasil, Teixeira, Lassance, Silva e Bardagi (2007) argumentam que falta aos pesquisadores um aprofundamento nos temas de referência na área; dentre eles a avaliação psicológica.

Sobre a produção científica em OPV nos Estados Unidos, Oliver, Lent e Zack (1998) apontam para o crescimento da pesquisa na área, ressaltando as contribuições oferecidas pela obra de Savickas e Walsh (1996), qual seja, Handbook of Career Counseling Theories and Practice, com vistas à reflexão sobre a desejada integração entre teoria e prática de Orientação Profissional. Nesse particular, vale destacar a ênfase de Oliver, Lent e Zack (1998) sobre as proposições de Gottfredson (1996), na direção de que uma única teoria não abarca todas as necessidades prementes na avaliação de carreira e sugere que sejam usados diferentes enfoques, de modo a enriquecer a testagem de modelos teóricos por meio de pesquisas empíricas. De modo complementar, Guindon e Richmond (2005) sugerem que ainda é grande a necessidade de integração entre teoria e prática em OPV.

No que diz respeito às investigações sobre interesses e outros construtos importantes em processos de OPV, Darcy e Tracey (2007) apontam que, normalmente, elas são realizadas em separado, ou seja, analisam-se interesses, personalidade e habilidades como literaturas distintas. Em contrapartida, são poucos os estudos que integram essas informações, que se preocupam em explicar e predizer o desenvolvimento de carreira e a escolha profissional. Os autores defendem que, ao somar essas informações, os profissionais poderão diminuir o uso de instrumentos que fornecerão dados redundantes e terão maior propriedade ao tentar integrar os resultados obtidos.

Em consonância com as proposições anteriores, Savickas (1995) conceitua interesses como uma forma de expressão da personalidade, uma vez que eles fazem a intermediação entre o indivíduo e o mundo do trabalho. Assim, ao avaliar os interesses e reconhecê-los como indicadores de personalidade, é possível obter informações sobre as necessidades, valores e preferências dos clientes. Esse autor afirma que interesse, no latim, significa "entre ser/estar". O construto representa o elo entre o indivíduo e o mundo, e, dessa forma, os interesses favoreceriam a prática de ajustamento às condições ambientais, em um processo de "auto-completamento", à medida que ajudariam na manutenção da integridade do indivíduo. Desse modo, destaca-se a relevância de se estudar esse construto em processos de OPV, uma vez que a ampliação do autoconhecimento sobre o tema pode ajudar os clientes a planejar seu futuro profissional, de modo a encontrar soluções que adaptem suas necessidades às oportunidades oferecidas pelo ambiente.

Um segundo grupo procura relacionar personalidade, motivação e interesses, compreendendo que os últimos estariam a serviço da personalidade. Mais especialmente, os interesses colaborariam com o desenvolvimento satisfatório da personalidade. Dentre os teóricos com maior destaque, convém mencionar Carter (1940), que defendia que os interesses promoviam a base para a integração da personalidade, ou Bodin (1943), que considerava o interesse como a expressão de si mesmo e do autoconceito ocupacional. Em comum, esses teóricos promulgaram a interpretação de que os interesses podem ser vistos como reflexo do autoconceito e da personalidade. Ainda nesse grupo encontram-se outros autores, como Holland (1963). Ele acreditava que os interesses expressavam características de personalidade em atividades de trabalho. Um dos instrumentos usados na presente pesquisa adota o referencial teórico de Holland (1963), que é a versão brasileira do SelfDirected Search - SDS.

O terceiro grupo reconhece a relevância do papel social. Assim, a aceitação ou rejeição ao papel social gera o gosto ou o desgosto por atividades ocupacionais; como referência, Savickas (1995) cita Tyler (1951). No tocante à última concepção, a imagem da ponte que une o papel social ao papel individual tem sido usada nessa definição. Nessa mesma direção, embora Gottfredson (1981) não tenha explicado a origem de interesses, enfatizava a importância de se estudar os interesses à luz de variáveis como raça, sexo, e nível socioeconômico. 
No que se refere aos estudos que investigaram os construtos mais comumente usados no contexto da OPV (Melo-Silva, 1999), serão destacados os que se seguem por se relacionarem com o tema do presente estudo. Com o objetivo de investigar a relação entre interesses, inteligência e personalidade, Ackerman e Heggestad (1997) realizaram uma extensa meta-análise das correlações entre habilidade intelectual, personalidade e interesses. Eles revisaram 135 estudos, tendo sugerido como resultado a existência de "complexos de traços", que integravam aspectos dos construtos. Em outras palavras, os "complexos de traços" são combinações de conceitos que destacam mais a comunalidade entre eles que aplicações específicas que eles possam ter. Posteriormente, na mesma direção da pesquisa anterior, Ackerman e Beier (2003) criticaram a prática de realização de intervenções que consideram interesses, personalidade e habilidades isoladamente.

Os "complexos de traços" foram detalhadamente descritos pelos autores como quatro agrupamentos, denominados Social, Convencional, Ciência/ Matemática e Intelectual/ Cultural. O primeiro grupo constituiu-se pelos interesses empreendedores e sociais, juntamente com os traços de personalidade extroversão, potência social e bem-estar subjetivo. Já o segundo envolvia a velocidade perceptual (medida de habilidade), interesses convencionais e personalidade com traços de controle, conscienciosidade/ realização e tradicionalismo. $\mathrm{O}$ terceiro, por sua vez, apresentava as habilidades de raciocínio matemático e percepção visual, aliados aos interesses realistas e investigativos. Por último, o quarto grupo formou-se pelos interesses investigativos $\mathrm{e}$ artísticos, pelas habilidades gc e fluência de idéias e pelos traços de personalidade absorção às atividades, abertura às experiências e engajamento intelectual típico (Ackerman \& Beier, 2003).

No Brasil, Primi e cols. (2002) estudaram a relação entre esses construtos com 60 clientes de orientação profissional, na sua maioria, jovens. Os autores usaram a Bateria de Provas de RaciocínioBPR-5, o Questionário de Personalidade 16-PF e o Levantamento de Interesses Profissionais (LIP). Nessa pesquisa, os autores estudaram as relações dos pares de construtos, ou seja, interesses e personalidade, interesses e habilidades, e habilidades e personalidade. Quanto aos resultados, verificaramse poucas correlações significativas entre habilidades e interesses (14 das 48 possíveis), sendo que, quando significativas, elas variaram entre $-0,25$ e 0,40 . De modo semelhante, ao analisar a relação entre personalidade (considerando os fatores secundários do 16-PF) e habilidades, houve apenas três correlações significativas, das 30 possíveis. Essas correlações foram de $0,25,0,26$ e 0,31 , sugerindo uma fraca relação entre os construtos. Por fim, houve algumas correlações significativas entre interesses e personalidade (seis das 30 possíveis), com magnitudes variando entre $-0,32$ e 0,42 , o que denota maior relação entre os construtos. O fator de personalidade que mais frequentemente se correlacionou significativamente com os interesses foi o Fator III (rigidez de pensamento), que indica comportamentos que vão de um contínuo de inflexibilidade, firmeza e baixa empatia a condutas de receptividade e abertura.

Por sua vez, Sagiv (2002) discute que os inventários de interesse, embora muito usados na prática de OPV, têm sérias limitações, especialmente no seu potencial para caracterizar os indivíduos em face de suas decisões. Para o autor, há outras variáveis que devem ser analisadas nesses momentos, dentre elas, os valores do trabalho, compreendidos como representações sociais e cognitivas das metas motivacionais, as quais servem para orientar os princípios de vida dos indivíduos. Em face disso, o autor investigou a relação entre valores e interesses em 97 pessoas de 19 a 54 anos (média 25,8), dos quais $77 \%$ tinha 12 anos de escolarização e $60 \%$ era mulher. Os participantes responderam ao SDS e a um instrumento de 57 itens, baseado nas proposições de Schwartz (1992), intitulado Portraits Questionnaire $\mathrm{PQ}$, para avaliar os valores associados às profissões.

Nessa pesquisa, os interesses e os valores correlacionaram-se sistematicamente, considerando grupos distintos (homens e mulheres), porém de forma moderada (todos com $p<0,05$ ). Mais especificamente, os interesses convencionais correlacionaram-se positivamente com valores de conformidade $(r=0,26)$, tradição $(r=0,21)$ e segurança $(r=0,40)$, e negativamente com os valores de autodirecionamento $(r=-0,35)$, estimulação $(r=-0,28)$ e universalismo $(r=-0,23)$. Por sua vez, os interesses empreendedores correlacionaram-se positivamente com os valores de poder $(r=0,40)$ e conquista $(r=0,32)$, e negativamente com universalismo $(r=-0,30)$. Os interesses sociais correlacionaram-se positivamente com o valor benevolência $(r=0,30)$, enquanto os interesses artísticos correlacionaram-se positivamente com os valores de auto-direcionamento $(r=0,28)$ e universalismo $(r=0,27)$, e negativamente com os valores de conformidade $(r=-0,22)$ e segurança $(r=$ $0,32)$. O interesse investigativo correlacionou-se apenas com o valor universalismo $(r=0,26)$ e apenas o tipo realista não se relacionou significativamente com nenhum dos valores básicos. Esses resultados permitem verificar associações existentes entre interesses e valores, sendo que esses últimos são 
compreendidos por alguns teóricos como parte das características de personalidade (exemplo Betz \& Borgen, 2000).

Com uma proposta semelhante, Armstrong, Smith, Donnay e Rounds (2004) aplicaram o Basic Interest Scale em 31.010 mulheres e 32.421 homens, com vistas a prover um sistema de classificação e um mapa de ocupações. Por meio da análise de clusters, foi possível encontrar nove interesses gerais, subdivididos em 19 áreas ocupacionais específicas. O mapa das representações permitiu identificar três dimensões chamadas persuasão versus solução de problemas; dinâmico versus estruturado; e serviço social versus trabalho solitário. Esse tipo de trabalho permite verificar aproximações entre as definições de interesses e traços de personalidade, uma vez que ambos os construtos descrevem as formas mais típicas das pessoas pensarem e agirem, ainda que os interesses $O$ façam de maneira mais restrita, considerando especificamente o contexto do trabalho.

Considerando a relevância de estudar as relações e possíveis sobreposições entre interesses profissionais, traços de personalidade e habilidades cognitivas para a teoria e prática de OPV, o presente estudo objetivou analisar as inter-relações entre esses construtos com adolescentes em processo de OPV. Esse tipo de estudo mostra-se pertinente também para a área de Avaliação Psicológica, considerando que esses três construtos encontram-se entre os mais pesquisados, conforme trabalhos apresentados nos Congressos de Avaliação Psicológica no Brasil (Joly \& cols., 2007). Ressalta-se, ainda, que esse tipo de estudo poderá auxiliar nas tentativas de integração entre teoria e prática em OPV e contribuir para a análise da informação diferencial provida por testes psicológicos em processos de avaliação psicológica tento em vista ampliar o autoconhecimento, quando da realização da escolha profissional de adolescentes do Ensino Médio.

\section{Método}

\section{Participantes}

Participaram da pesquisa 211 alunos do ensino médio de escola particular do estado do Paraná. A maioria era mulher $(58,3 \%)$, com idade variando entre 16 e 18 anos (média 16,5; DP 0,5 anos). Quanto à condição socioeconômica, $51,1 \%$ informou pertencer a famílias com renda mensal superior a 15 salários mínimos (SM); 41,5\% tinha renda entre 5 e $15 \mathrm{SM}$ e apenas $7,3 \%$ tinha renda entre 1 e 5 SM. A maioria dos jovens $(96,9 \%)$ indicou ter sido criada em área urbana, e no momento da coleta também residia em área urbana.

\section{Instrumentos}

Foram utilizados quatro testes, a saber, Bateria de Provas de Raciocínio (BPR-5) (Primi \& Almeida, 2000), Bateria Fatorial de Personalidade (BFP) (Nunes, Hutz \& Nunes, no prelo), Escala de Aconselhamento Profissional (EAP) (Noronha, Sisto \& Santos, 2007) e Self Directed Search (SDS) (Primi, Mansão, Muniz \& Nunes, no prelo). Esses testes foram escolhidos por utilizarem referenciais teóricos bastante pesquisados, a saber, a tipologia de Holland, o modelo dos Cinco Grandes Fatores de Personalidade e o modelo de inteligência CHC Cattel-Horn-Carroll, além de serem instrumentos que já contam com evidências de validade e precisão favoráveis ao seu uso. Quanto ao EAP, os autores fazem referência à definição de Savickas (1995), como a que melhor representa o instrumento. Os testes serão descritos a seguir.

A BPR-5 é uma bateria para avaliação de habilidades cognitivas. Presentemente foram usadas quatro provas, especificamente Raciocínio Verbal, Raciocínio Abstrato, Raciocínio Numérico e Raciocínio Espacial. As provas avaliam, respectivamente, extensão e profundidade do conhecimento verbal vocabular; capacidade de resolver problemas com estímulos abstratos; conhecimento de equações simples de matemática/ habilidade quantitativa; e capacidade de resolver problemas que exigem processamento mental de imagens. Essa bateria possui evidências de validade e precisão descritas em seu manual, sendo um teste que possui parecer favorável pelo Conselho Federal de Psicologia, 2008. As respostas são corrigidas como certas ou erradas e o escore das provas é gerado pela soma dos acertos. Foi utilizada a forma B da bateria, indicada para alunos do ensino médio (Primi \& Almeida, 2000).

A Bateria Fatorial de Personalidade avalia a personalidade por meio do modelo dos Cinco Grandes Fatores. A escala possui 126 itens, respondidos em escala likert de 7 pontos, na qual o sujeito indica a concordância com as afirmativas, que descrevem a expressão da personalidade por meio da forma como as pessoas pensam, agem e sentem. A BFP avalia os fatores neuroticismo, socialização, realização, abertura e extroversão. Extroversão referese à quantidade e à intensidade das interações interpessoais preferidas, nível de atividade, necessidade de estimulação e capacidade de alegrarse. Socialização também é uma dimensão interpessoal, porém avalia o interesse pelo bem-estar dos outros, confiança nas pessoas e adesão a regras ou normas sociais (Nunes e cols., no prelo).

Realização representa o grau de organização, 
persistência, controle e motivação para alcançar objetivos, avaliando especificamente o senso de competência pessoal, a ponderação e o comprometimento na busca de objetivos. Por sua vez, neuroticismo refere-se ao nível crônico de ajustamento emocional e instabilidade, envolvendo especificamente a vulnerabilidade à opinião dos outros, instabilidade de humor/emocional, presença de sintomas depressivos e comportamentos passivos ou falta de energia para agir em situações importantes. Por fim, o fator abertura para novas experiências refere-se aos comportamentos exploratórios e de reconhecimento da importância de ter novas experiências, sendo que esse fator avalia interesse por novas idéias, liberalismo e busca por novidades (Nunes e cols., no prelo).

A BFP encontra-se em análise no Conselho Federal de Psicologia, de modo que serão descritas algumas informações adicionais neste artigo, enquanto o manual ainda não está disponível para uso de psicólogos. As evidências de validade da BFP foram investigadas a partir de uma base de dados com 6.599 pessoas, com idade média de 23,7 anos $(\mathrm{DP}=9,6)$, que participaram de 18 estudos distintos, de 11 estados brasileiros. As evidências de validade envolveram a análise da estrutura interna, a correlação com outras variáveis (convergentedivergente) e a análise de critérios externos. A precisão por alfa de Cronbach foi de 0,89 (neuroticismo), $\quad 0,84 \quad$ (extroversão), 0,85 (socialização), 0,83 (realização) e 0,74 (abertura).

A Escala de Aconselhamento Profissional avalia preferências ou interesses profissionais por meio de sete dimensões, denominadas Ciências Exatas, Artes e Comunicação, Ciências Biológicas e da Saúde, Ciências Agrárias e Ambientais, Atividades Burocráticas, Ciências Humanas e Sociais aplicadas, e Entretenimento. A escala possui 61 itens que medem a preferência por atividades profissionais, com opção de resposta de 1 a 5 em escala likert, referindo o quanto a pessoa gostaria de realizar as atividades descritas. Ciências Exatas tem itens associados a atividades de desenvolvimento de tecnologia e ao uso e desenvolvimento de aparelhos eletrônicos ou de softwares. Já Artes e Comunicação envolve atividades que usam a criatividade, tais como criação de textos ou envolvimento com teatro, entre outros (Noronha e cols., 2007).

Por sua vez, a dimensão Ciências Biológicas e da Saúde contém itens que envolvem o cuidado das pessoas com um caráter mais assistencialista, em situações nas quais os pacientes estão doentes ou apresentam patologias, destacando o gosto por ajudar os outros. Ciências Agrárias e Ambientais avalia o cuidado do meio ambiente, o desenvolvimento de ações para preservar e monitorar a natureza e a preferência por trabalho em locais abertos. As Atividades Burocráticas retratam situações que envolvem a organização e classificação de informações, além de itens sobre o contato com pessoas por meio de situações de seleção de pessoal ou de negociação entre empresa e empregados. As Ciências Humanas e Sociais indicam o gosto de conhecer e estudar o comportamento humano, analisar questões sociais e culturais, além de atividades de organização de materiais. Por último, a dimensão Entretenimento destaca o caráter de trabalho com o público no sentido de promover o bem-estar por meio do lazer e diversão, em atividades com turistas, com moda ou publicidade (Noronha e cols., 2007). A EAP possui parecer favorável pelo Conselho Federal de Psicologia, 2008.

O SDS avalia seis tipos de interesses, de acordo com a tipologia de Holland, conhecida pela sigla RIASEC. O teste possui 216 itens, divididos em quatro seções. No Brasil, sua estrutura interna foi avaliada, indicando a existência das seis dimensões esperadas teoricamente, além de ter sido encontrada evidência de validade convergente, por meio da correlação com outros testes de interesse, e divergente, por meio da correlação com as provas do BPR-5. A precisão medida pelo alfa de Cronbach variou entre 0,87 e 0,90 , e por teste-reteste, entre 0,82 e 0,91 (Mansão \& Yoshida, 2006). Quanto à descrição do conteúdo psicológico avaliado pelos seis tipos de interesse, o realista envolve interesse por atividades com elementos concretos, o uso da força física e o contato com a natureza. $O$ investigativo gosta mais de atividades intelectuais, que utilizam a razão e a curiosidade, com tendência à maior introspecção e abstração de conceitos. O artístico destaca-se pelo gosto do uso da sensibilidade e da criatividade, não privilegiando atividades concretas ou burocráticas. O social apresenta interesse por tarefas assistenciais, de cuidado e de busca pelo bem-estar do outro. O empreendedor destaca-se pela capacidade de argumentação, bom contato social e evitação de tarefas repetitivas. Por fim, o convencional envolve-se mais em atividades burocráticas, o trabalho com finanças e o gosto por tarefas repetitivas (Holland, Fritzsche \& Powell, 1994).

\section{Procedimentos}

Os testes foram aplicados coletivamente, em grupos de aproximadamente 20 jovens, em processo de OPV. O processo durou dois meses, com foco na ampliação do autoconhecimento. Os jovens responderam um teste por sessão, no caso do SDS, 
EAP e BFP, sendo que, em relação ao BPR-5, duas provas foram aplicadas por sessão. Para a realização da pesquisa, os responsáveis pelos jovens assinaram o Termo de Consentimento Livre e Esclarecido.

Quanto à análise de dados, inicialmente os escores brutos dos fatores de personalidade, interesses e de inteligência foram convertidos em escore $z$, em devido suas métricas desiguais. Posteriormente, procedeu-se a uma análise dos componentes principais com rotação varimax para os fatores dos testes, a saber: os cinco fatores de personalidade, os quatro escores de habilidade, os seis tipos de interesse do SDS e as sete dimensões de preferências ocupacionais da EAP. Desse modo, a análise contou com 22 variáveis a serem analisadas, considerando 211 pessoas, ou seja, com uma proporção aproximada de 9,5 pessoas por fator analisado.

\section{Resultados}

Primeiramente analisou-se o KMO, que foi de 0,66 e o Teste de Bartlett, que foi significativo $(p<0,001)$. Apesar do KMO ter sido relativamente baixo, o Teste de Bartlett foi significativo, o que sugeriu a possibilidade de extração de fatores. Para uma análise inicial da configuração dos agrupamentos/dimensões possíveis entre as variáveis dos testes, observou-se o gráfico scree plot, conforme Figura 1.

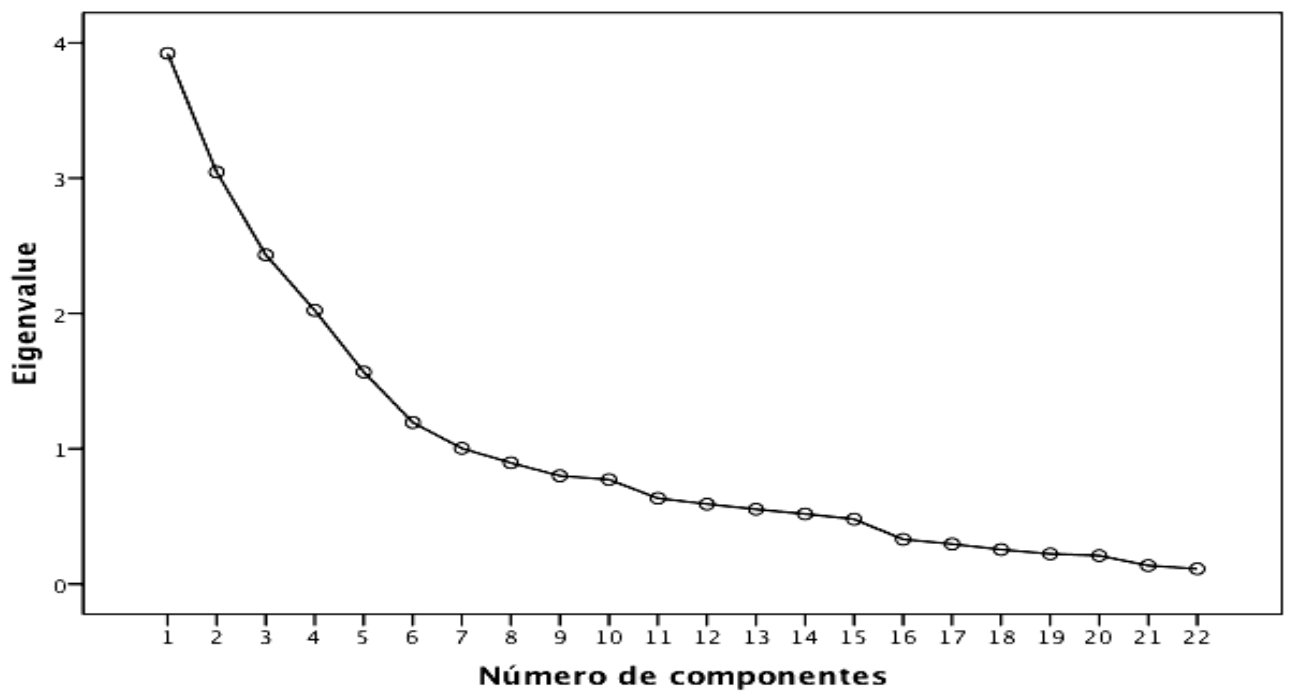

Figura 1 - Gráfico scree da análise dos interesses, personalidade e inteligência

Por meio da análise do scree plot considerou-se a extração de seis ou sete fatores. Quanto aos autovalores, sete fatores apresentaram índices superiores a 1. Foram analisados os conteúdos das soluções com seis e sete fatores, porém a solução de seis fatores apresentou maior coerência teórica. Essa solução explicou $64,5 \%$ da variância, com fatores com autovalores superiores a 1,5. Foi adotada como linha de corte para a consideração das cargas fatoriais o patamar de 0,30 e o método de rotação varimax. A matriz de componentes é apresentada na Tabela 1. Destaca-se que, para facilitar a compreensão da Tabela, ao lado do nome de cada fator original dos testes (exemplo: tipo social, neuroticismo, etc.), acrescentou-se o nome do teste a que o fator pertence e o construto que é avaliado.

Tabela 1 - Matriz de componentes da análise dos componentes principais entre inteligência, interesses e personalidade

Continua

\begin{tabular}{|c|c|c|c|c|c|c|}
\hline Fatores & 1 & 2 & 3 & 4 & 5 & 6 \\
\hline Artes e comunicação - EAP - Interesses & 0,86 & & & & & \\
\hline Artístico - SDS - Interesses & 0,84 & & & & & \\
\hline Abertura - BFP - Personalidade & 0,74 & & & & & \\
\hline Ciências humanas e sociais aplicadas - EAP - Interesses & 0,58 & 0,45 & 0,32 & & & \\
\hline Atividades burocráticas - EAP - Interesses & & 0,84 & & & & \\
\hline
\end{tabular}


Tabela 1 - Matriz de componentes da análise dos componentes principais entre inteligência, interesses e personalidade

\begin{tabular}{|c|c|c|c|c|c|c|}
\hline Fatores & 1 & 2 & 3 & 4 & 5 & 6 \\
\hline Empreendedor - SDS - Interesses & & 0,81 & & & & \\
\hline Convencional - SDS - Interesses & & 0,77 & & & & \\
\hline Entretenimento - EAP - Interesses & 0,45 & 0,61 & & & & \\
\hline Ciências biológicas e da saúde- EAP - Interesses & & & 0,76 & & & \\
\hline Ciências agrárias e ambientais- EAP - Interesses & & & 0,68 & 0,37 & & \\
\hline Social- SDS - Interesses & & & 0,68 & & & \\
\hline Socialização - BFP - Personalidade & & & 0,63 & & & \\
\hline Ciências exatas - EAP - Interesses & & & & 0,83 & & \\
\hline Realista- SDS - Interesses & & & & 0,74 & & \\
\hline Investigativo - SDS - Interesses & & & 0,53 & 0,67 & & \\
\hline Raciocínio espacial - BPR-5 - Inteligência & & & & & 0,74 & \\
\hline Raciocínio numérico- BPR-5 - Inteligência & & & & & 0,71 & \\
\hline Raciocínio verbal- BPR-5- Inteligência & & & & & 0,64 & \\
\hline Raciocínio abstrato - BPR-5 - Inteligência & & & & & 0,60 & \\
\hline Neuroticismo - BFP - Personalidade & & & & & & 0,75 \\
\hline Extroversão - BFP - Personalidade & 0,36 & & & & & 0,67 \\
\hline Realização - BFP - Personalidade & & & & & & 0,45 \\
\hline Precisão dos fatores (Alfa de Cronbach) & 0,80 & 0,79 & 0,69 & 0,74 & 0,61 & 0,34 \\
\hline
\end{tabular}

Os quatro primeiros fatores mesclaram aspectos de interesses e personalidade. O primeiro fator englobou um conteúdo que envolve o interesse por trabalhos que exigem criatividade (tipo artístico e dimensão artes e comunicação), a quebra de padrões preestabelecidos, o gosto por descobrir coisas novas (fator de personalidade abertura) e o interesse por questões do comportamento humano e culturais (dimensão ciências humanas e sociais aplicadas). Destaca-se que essa última dimensão da EAP teve carga superior a 0,30 em três fatores, sugerindo que esse interesse deve representar um padrão de gosto por atividades presentes em diferentes áreas profissionais. $\mathrm{O}$ primeiro fator também foi acompanhado pelo traço de personalidade extroversão e pelo interesse na área de entretenimento, ainda que em ambos os casos tenham apresentado carga fatorial baixa, sugerindo o gosto por contatos sociais, dinamismo, valorização do próprio trabalho e bom nível de comunicação, no que se refere à personalidade, e o interesse por trabalhos de lazer e diversão destinados e clientes como turistas.

$\mathrm{O}$ segundo fator encontrado envolveu, por um lado, o interesse por tarefas rotineiras (tipo convencional e dimensão atividades burocráticas) e, por outro, o gosto por atividades que envolvem liderança e comunicação (tipo empreendedor) e o contato com o público para promover situações de lazer (dimensão entretenimento). Por sua vez, o terceiro fator englobou aspectos relativos ao desejar o bem-estar aos outros, com ações de cunho assistencial ou de ensino (tipo social), de cuidado na área da saúde (dimensão ciências biológicas e da Saúde), da preservação da natureza (dimensão ciências agrárias e ambientais) e ações de ajuda e de preocupação com os outros, de confiança nos demais e de gosto pelo cumprimento de regras sociais (traços de personalidade de socialização). $O$ terceiro fator teve ainda a participação do tipo investigativo do SDS, acrescentando o gosto pela pesquisa e pela resolução de problemas complexos.

$\mathrm{O}$ quarto fator pode ser interpretado como o gosto por trabalhos com elementos concretos e objetivos (tipo realista), o uso de tecnologia (dimensão de ciências exatas) e o gosto por pesquisa e por trabalhos introspectivos (tipo investigativo). Adicionalmente, é possível que o interesse por elementos concretos e que não privilegiam o contato social também esteja acompanhados pela dimensão ciências agrárias e ambientais, ainda que com carga 
fatorial baixa, o que aponta para o gosto pelo trabalho com elementos do meio ambiente $\mathrm{e}$ desenvolvimento de tecnologia para a preservação do mesmo.

Por fim, a análise da estrutura interna permitiu observar que o $5^{\circ}$ e $6^{\circ}$ fatores foram compostos, respectivamente, apenas por provas de inteligência, e apenas por fatores de personalidade. Desse modo, foi possível observar a contribuição única da avaliação da inteligência, assim como dos fatores neuroticismo e realização, que não revelaram relação forte com nenhum tipo de interesse ou habilidade cognitiva. A precisão dos quatro primeiros fatores foi superior a 0,69 e dos dois últimos, respectivamente, 0,61 e 0,34.

\section{Discussão}

Esse estudo destinou-se à investigação das relações entre três construtos comumente usados em processo de OPV, como identificado, por exemplo, por Joly e cols. (2007) e Noronha e Nunes (2008): inteligência, personalidade e interesses. Mais especificamente, pretendeu-se observar o padrão de correlações desses construtos. Os resultados pareceram bastante promissores. Do total de 22 variáveis, foi obtida uma organização satisfatória em seis conjuntos, sendo que a inteligência e alguns dos fatores de personalidade não se inseriram nos relacionados aos interesses. Destaca-se que as habilidades cognitivas não tiveram associação forte com os fatores de personalidade e de interesses, o que parece justificar a manutenção da análise da inteligência em processos de OPV, uma vez que os outros domínios não trazem informação referente a esse aspecto. Quanto aos interesses, verificou-se comunalidades entre fatores do EAP e SDS, sugerindo que o uso de ambos os testes em um processo de OPV traz informações, em alguma medida, redundantes. Nesse específico, vale lembrar que a escolha do teste deverá ser guiada pelas características da clientela e demandas específicas que apresentam (Urbina, 2007).

Por sua vez, os traços de personalidade que mais se aproximaram dos padrões de interesse foram socialização, extroversão e abertura a experiências. Os fatores realização e neuroticismo apresentaram uma contribuição específica nessa análise, sugerindo não estar associado a interesses por profissões ou tipos de atividades específicas. $\mathrm{O}$ traço realização pode ser útil quando da condução de processos de OPV para refletir sobre o nível de determinação para atingir os objetivos, a possibilidade de fazer sacrifícios para alcançar o que deseja, a clareza sobre quais são as metas pessoais, a responsabilidade ante as tarefas que assume, entre outros, não sendo, desse modo, útil na análise relacionada a áreas profissionais particulares, mas sim, de uma forma geral. Já neuroticismo indica o grau de instabilidade emocional, níveis de ansiedade e depressão vivenciados, e vulnerabilidade à opinião e aos comentários de terceiros. Desse modo, esse fator pode ser mais bem aproveitado quando do questionamento sobre a capacidade geral das pessoas para a tomada de decisão, ou seja, aparentemente esse traço será mais útil quando relacionado, por exemplo, com as dificuldades de tomada de decisão profissional ou outros desajustamentos psicológicos associados à escolha (Saka, Gati \& Kelly, 2008).

Outra observação relevante é a aproximação de alguns tipos de Holland e de algumas áreas de interesse da EAP. Esses dados são especialmente úteis para orientadores que utilizam esses instrumentos, tendo em vista perceber as comunalidades entre tipos ou áreas de interesses, ao invés de analisá-las como aspectos separados. Assim, é possível pensar, por exemplo, que o fator 3, composto pelo tipo SocialSDS, pelas Ciências Biológicas e da Saúde-EAP, pelas Ciências Agrárias e Ambientais-EAP e pelo traço de personalidade Socialização-BFP, revela em comum o gosto pelas interações sociais, com foco na realização de ações de ajuda, de promoção do bem-estar dos outros, porém com áreas de aplicação distintas. Em outras palavras, algumas profissões envolvem o cuidado físico, outras, o psicológico, outras, o cuidado do meio ambiente, e assim por diante.

Ressalta-se que as dimensões Ciências Humanas e Sociais Aplicadas, Entretenimento, Ciências Agrárias e Ambientais, o tipo Investigativo e o traço de personalidade Extroversão foram os elementos que estiveram presentes em mais de um fator. Esse dado pode ser interpretado no sentido de que esses são padrões de gosto ou formas mais típicas de pensar e agir que estão presentes em mais áreas profissionais. Outro comentário pertinente é que alguns fatores da EAP são compostos por itens que participam de mais de um fator, o que favorece a configuração presentemente observada. Considerando esse resultado, outras pesquisas poderiam verificar qual a contribuição única de cada dimensão na reflexão sobre a escolha da profissão, pois talvez esses sejam elementos amplos para o uso em OPV.

Os achados presentemente encontrados não estão completamente em consonância com os de Ackerman e Beier (2003), uma vez que, dentre os quatro conjuntos encontrados pelos autores, apenas o último (interesses investigativos e artísticos; habilidades $g c$ e fluência de idéias; e traços de personalidade absorção às atividades, abertura às experiências e engajamento intelectual típico) se aproximou de um dos fatores revelados nessa análise 
(artístico - SDS; Artes e comunicação - EAP; abertura - BFP; ciências humanas e sociais aplicadas EAP; entretenimento - EAP e extroversão - BFP). As diferenças observadas podem se dever aos instrumentos utilizados nos dois estudos e aos respectivos referenciais teóricos empregados, que não são os mesmos do presente estudo; além das especificidades das amostras. Nesse particular, seria interessante a realização de pesquisas no Brasil com testes que utilizassem o mesmo referencial teórico de Ackerman e Beier (2003), de modo a testar se o modelo é aplicável à nossa realidade.

Já as conclusões de Primi e cols. (2002), embora tenham se valido de um estudo correlacional, estiveram mais próximas deste estudo. Nesse sentido, os autores identificaram baixas relações entre habilidades e interesses, corroborando a configuração das provas de inteligência em um único fator. De modo semelhante, ao analisar a relação entre personalidade e habilidades, foram encontradas apenas três correlações significativas, sendo que, presentemente, a análise fatorial de segunda ordem revelou que, dos cinco fatores de personalidade, dois se agruparam em um único fator, enquanto os outros três se juntaram aos demais fatores de interesses.

Quanto à análise da consistência interna dos fatores extraídos, a precisão dos quatro primeiros fatores pode ser considerada satisfatória, enquanto o quinto teve uma precisão regular e o sexto, baixa (Hair, Tatham \& Black, 2005). No entanto, o fato de as provas de inteligência apresentarem baixa precisão não parece comprometer o presente estudo, uma vez que avaliam habilidades distintas. Da mesma forma, os fatores de personalidade não possuem um fator comum, o que por si só sugere que a precisão seria baixa.

\section{Considerações finais}

Tal como já destacado, a análise da relação entre interesses, personalidade e habilidades pareceu relevante para ampliar o conhecimento sobre alguns testes que podem ser utilizados em avaliações psicológicas com foco em Orientação Profissional/ Vocacional (OPV). Contudo, os dados observados necessitam de mais investigações, considerando as divergências apresentadas em relação a alguns estudos (exemplo Ackerman \& Beier, 2003), bem como a complexidade da reflexão que se apresenta. Muitos são os elementos que necessitam de consideração, como as amostras, os instrumentos, as diversidades culturais e os construtos teóricos.

Presentemente defende-se a análise integrada dos interesses, personalidade e habilidades, uma vez que considera-se que essa perspectiva possibilitará um aprimoramento da qualidade da informação provida aos clientes que buscam ampliar $\mathrm{O}$ autoconhecimento, ao auxiliá-lo a pensar sobre aspectos relacionados à escolha da profissão. No entanto, é importante retomar as considerações da APA, AERA e NCME, 1999 sobre a importância da escolha adequada dos instrumentos para a avaliação psicológica, ao evitar que os clientes realizem extensas avaliações desnecessariamente. Desse modo, para a análise dos interesses, por exemplo, é importante uma reflexão sobre qual instrumento melhor se adapta às necessidades do cliente, não sendo recomendado, a priori, o uso de dois testes, uma vez que eles podem apresentar informações parcialmente redundantes, tal como observado na presente pesquisa.

Em síntese, trabalhos dessa natureza devem ganhar espaço nas pesquisas científicas, já que a compreensão das relações entre os fenômenos psicológicos investigados tende a colaborar com os processos de OPV, como já havia sido enfatizado por Ackerman e Heggestad (1997) e por Darcy e Tracey (2007). À medida que isto estiver mais frequente, além de favorecer o aprimoramento dos processos de OPV, será possível ainda, atender às solicitações de Savickas e Walsh (1996) e Guindon e Richmond (2005), na direção da integração entre teoria e prática na área.

A título de finalização, recomenda-se que os estudos sobre a temática continuem em foco, de modo que gerem dados e reflexões a respeito de instrumentos e resultados. Ainda, quanto ao presente estudo, recomenda-se cautela na generalização, uma vez que trabalhou uma amostra restrita.

\section{Referências}

Ackerman, P. L. \& Heggestad, E. D. (1997). Inteligence, personality and interests: evidence for overlapping traits. Psychological Bulletin, 121, 219-245.

Ackerman, P. L. \& Beier, M. E. (2003). Intelligence, personality, and interests in the career choice process. Journal of Career Assessment, 11 (2), 205218.

American Educational Research Association, American Psychological Association \& National Council on Measurement in Education. (1999). Standards for educational and psychological testing. Washington, DC: Autor.

Armstrong, P. I., Smith, T. J., Donnay, D. A. C. \& Rounds, J. (2004). The strong ring: A basic 
interest model of occupational structure. Journal of Counseling Psychology, 51(3), 299-313.

Betz, N. E. \& Borgen, F. H. (2000). The future of career assessment: integrating vocational interests with self-efficacy and personal styles. Journal of Career Assessment, 8(4), 329-338.

Conselho Federal de Psicologia - CFP. (2008). SATEPSI - Lista dos testes aprovados. Obtido em 20 de janeiro de 2008 do world wide web: http://www.pol.org.br.

Darcy, M. \& Tracey, T. J. G. (2007). Integrating abilities and interests in career choice: maximal versus typical assessment guindon. Journal of Career Assessment, 11(2), 219-237.

Guindon, M. H. \& Richmond, L. J. (2005). Practice and Research in Career Counseling and Development - 2004. The Career Development Quarterly December, 54, 90-137.

Hair, J. F., Tatham, R. L. \& Black, W. C. (2005). Análise multivariada de dados. Porto Alegre: Bookman.

Holland, J. L., Fritzsche, B. A. \& Powell, A. B. (1994). SDS- Self - Directed Search. Los Angeles, California: PAR - Psychological Assessment Resources.

Joly, M. C. A. J., Silva, M. C. R., Nunes, M. F. O., \& Souza, M. S. (2007). Análise da produção científica em painéis dos Congressos Brasileiros de Avaliação Psicológica. Avaliação Psicológica, 6(2), 239-252.

Mansão, C. S. M. \& Yoshida, E. M. P. (2006). SDS: Questionário de Busca Autodirigida - precisão e validade. Revista Brasileira de Orientação Profissional, 7(2), 67-80.

Melo-Silva, L. (1999). A formação do orientador profissional. Revista da ABOP, 3(1), 161-165.

Noronha, A. P. P. \& Ambiel, R. A. M. (2006). Orientação profissional e vocacional: análise da produção científica. PsicoUsf, 11 (1), 75-84.

Noronha, A. P. P. \& Nunes, M. F. O. (2008). Produções no Congresso de Avaliação Psicológica no Brasil: Caminhos Traçados e Novos Desafios. Em A. P. P. Noronha, C. Machado, L. Almeida, M. Gonçalves, S. Martins \& V. Ramalho (Eds.). Actas da XIII Conferência Internacional de Avaliação Psicológica: formas $e$ contextos (pp. 1-14). Braga: Psiquilíbrios.

Noronha, A. P., Sisto, F., \& Santos, A. A. A. (2007). Escala de Aconselhamento Profissional - EAP manual técnico. Itatiba-SP: Vetor.
Noronha, A. P. P., Andrade, R. G., Miguel, F. K., Nascimento, M. M., Nunes, M. F. O., Pacanaro, S. V. e cols. (2006). Análise de teses e dissertações em orientação profissional. Revista da $A B O P, 7(2)$, $1-10$.

Nunes, C. H. S. S., Hutz, C. S., \& Nunes, M. F. O. (no prelo). Bateria Fatorial de Personalidade (BFP): manual técnico. Itatiba, SP: Casa do Psicólogo.

Oliver, L. W., Lent, E. B. \& Zack, J. S. (1998). Career and vocational assessment 1995-1996: a biennial review. Journal of Career Assessment, 6(3), 231-268.

Primi, R. \& Almeida, L. S. (2000). Bateria de Provas de Raciocinio (BPR-5). Manual técnico. São Paulo: Casa do Psicólogo.

Primi, R., Biguetti, C. A., Munhoz, A. M. H., Noronha, A. P., Polydoro, S., Nucci, E. P. D. \& Pelegrini, M. C. K. (2002). Personalidade, interesses e habilidades: um estudo correlacional da BPR-5, LIP e do 16-PF. Avaliação Psicológica, 1(1), 61-72.

Primi, R., Mansão, C. S. M., Muniz, M. \& Nunes, M. F. O. (no prelo). SDS - Questionário de Busca AutoDirigida - manual técnico da bersão brasileira. São Paulo: Casa do Psicólogo.

Sagiv, L. (2002). Vocational interests and basic values. Journal of Career Assessment, 10 (2), 233-257.

Saka, N., Gati, I. \& Kelly, K. R. (2008). Emotional and personality-related aspects of career decision making difficulties. Journal of Career Assessment, 16 (4), 403-424.

Savickas, M. L. (1995). Examining the personal meaning of inventoried interests during career Counseling. Journal of Career Assessment, 3(2), 188201.

Schwartz, S. H. (1992). Universals in the content and structure of values: theoretical advances and empirical tests in 20 countries. Em M. Zanna (Ed.), Advances in Experimental Social Psychology, Vol. 25 (pp. 1-65). New York: Academic Press.

Sparta, M., Bardagi, M. P., \& Teixeira, M. A. P. (2006). Modelos e Instrumentos de Avaliação em orientação profissional: perspectiva histórica e situação no Brasil. Revista Brasileira de Orientação Profissional, 2006, 7(2), 19-32.

Teixeira, M. A. P., Lassance, M. C. P., Silva, B. M. B. \& Bargaggi, M. P. (2007). Produção científica em orientação profissional: uma análise da Revista Brasileira de Orientação Profissional. Revista Brasileira de Orientação Profissional, 8(2), 25-40.

Urbina, S. (2007). Fundamentos da testagem psicológica. Porto Alegre: Artmed. 
Recebido em abril de 2008

Reformulado em outubro de 2008

Aprovado em dezembro de 2008

Sobre as autoras:

Maiana Farias Oliveira Nunes é psicóloga pela Faculdade Ruy Barbosa, mestre e doutoranda em Psicologia, com ênfase em Avaliação Psicológica pela Universidade São Francisco. Bolsista Capes, tem experiência com os temas Construção e Validação de Instrumentos Psicológicos, Orientação Profissional e Avaliação da Personalidade.

Ana Paula Porto Noronha é psicóloga e doutorada em psicologia,

Ciência e Profissão pela Pontifícia Universidade Católica de Campinas (1999). Atualmente é professora associada do Programa de Pós-Graduação Stricto Sensu da Universidade São Francisco. Tem experiência na área de Psicologia, com ênfase em Fundamentos e Medidas da Psicologia. É presidente atual do Instituto Brasileiro de Avaliação Psicológica e bolsista produtividade em pesquisa do CNPq. 